\title{
LETTER
}

\section{Spontaneous echo contrast in venous ultrasound of severe COVID-19 patients}

\author{
Siddharth Dugar ${ }^{1,2^{*}}$ (D) Abhijit Duggal ${ }^{1,2}$, Akbik Bassel $^{1}$, Mona Soliman ${ }^{1}$ and Ajit Moghekar ${ }^{1,2}$
}

(c) 2020 Springer-Verlag GmbH Germany, part of Springer Nature

\section{Dear Editor,}

Initial reports have indicated a higher incidence of venous thromboembolism (VTE) among patients with coronavirus disease 2019 (COVID-19) compared to other critical illnesses. Helms et al. [1] found pulmonary embolisms in $25 \%$ of patients undergoing CT pulmonary angiography despite prophylactic or therapeutic anticoagulation. Similarly, a $27 \%$ incidence of VTE was described by Klok et al. [2] in critically ill COVID19 patients despite use of standard thromboprophylaxis. Recently, Llitjos et al. [3] found a higher rate of VTE (69\%) among severe COVID-19 patients while on anticoagulation.

We confirm a 31\% incidence of VTE among patients with COVID-19 admitted to the ICU, and our group of experienced point of care ultrasound (POCUS) trained intensivists ( $>5$ years) noticed a striking pattern of spontaneous echo contrast (SEC) in the venous system during central line placements. This led us to develop a protocol for lower extremity POCUS scans on all COVID-19 patients in the ICU that included optimization of the gain till no SEC was observed in the adjacent arterial lumen. Lower extremity POCUS scan of 2-region compression ultrasonography can be carried out by intensivists rapidly and accurately [4].

In this study, POCUS was performed on 38 consecutive patients with COVID-19 admitted to our ICU (Fig. 1). We first excluded 7 patients; 4 with missing data and 3 with repeat scans. Of the remaining 31 patients, SEC was observed among 22 patients; however, the average time of scan was $3.93 \pm 3.71$ days with a significant difference in the SEC group ( $\mathrm{N}=22,2.75$ days \pm 2.3 days) compared to normal venous group $(\mathrm{N}=9,6.56$ days \pm 4.6 days). To adjust for time variability, an additional 13 patients, who were scanned beyond $72 \mathrm{~h}$, were excluded. Among the 18 patients analyzed in the study, 83\% demonstrated SEC in more than 2 sites (Image 1, Video 1). Notably, 87.5\% in the SEC group showed bilateral evidence in all sites. This finding was independently verified and confirmed by 2 physicians (S.D; A.M). All 18 patients were receiving anticoagulation $(\mathrm{N}=18$ on thromboprophylaxis, $\mathrm{N}=4$ on heparin infusions due to renal replacement therapy [RRT]). A concomitant echocardiogram was performed in $72 \%$ of patients among which $83 \%$ did not have abnormalities. Elevated inflammatory markers, a higher need for RRT (67\% vs 33\%) and an increased incidence of deep vein thrombosis (DVT; $40 \%$ vs $33 \%$ ), were detected in the SEC group as compared to those with normal venous flow (ESM Table 1).

Jensen et al. [5] have described amorphous echogenicity occupying the entire lumen of a fully compressible venous lumen, greater than the adjacent artery as a marker of venous stasis in oncology patients. They also correlated a qualitative SEC with lower peak venous velocities with a doubling in the incidence of DVT. In light of this, the SEC observed among severe COVID19 patients could likely indicate a precursor for VTE. As a result, higher intensity thromboprophylaxis may be appropriate for this group of SEC patients and future studies are investigating clinical outcomes with anticoagulation strategies.

*Correspondence: siddharthdugarmd@gmail.com

${ }^{1}$ Department of Critical Care Medicine, Respiratory Institute, Cleveland Clinic, 9500 Euclid Ave, Cleveland, OH 44195, USA

Full author information is available at the end of the article

\section{实 Springer}




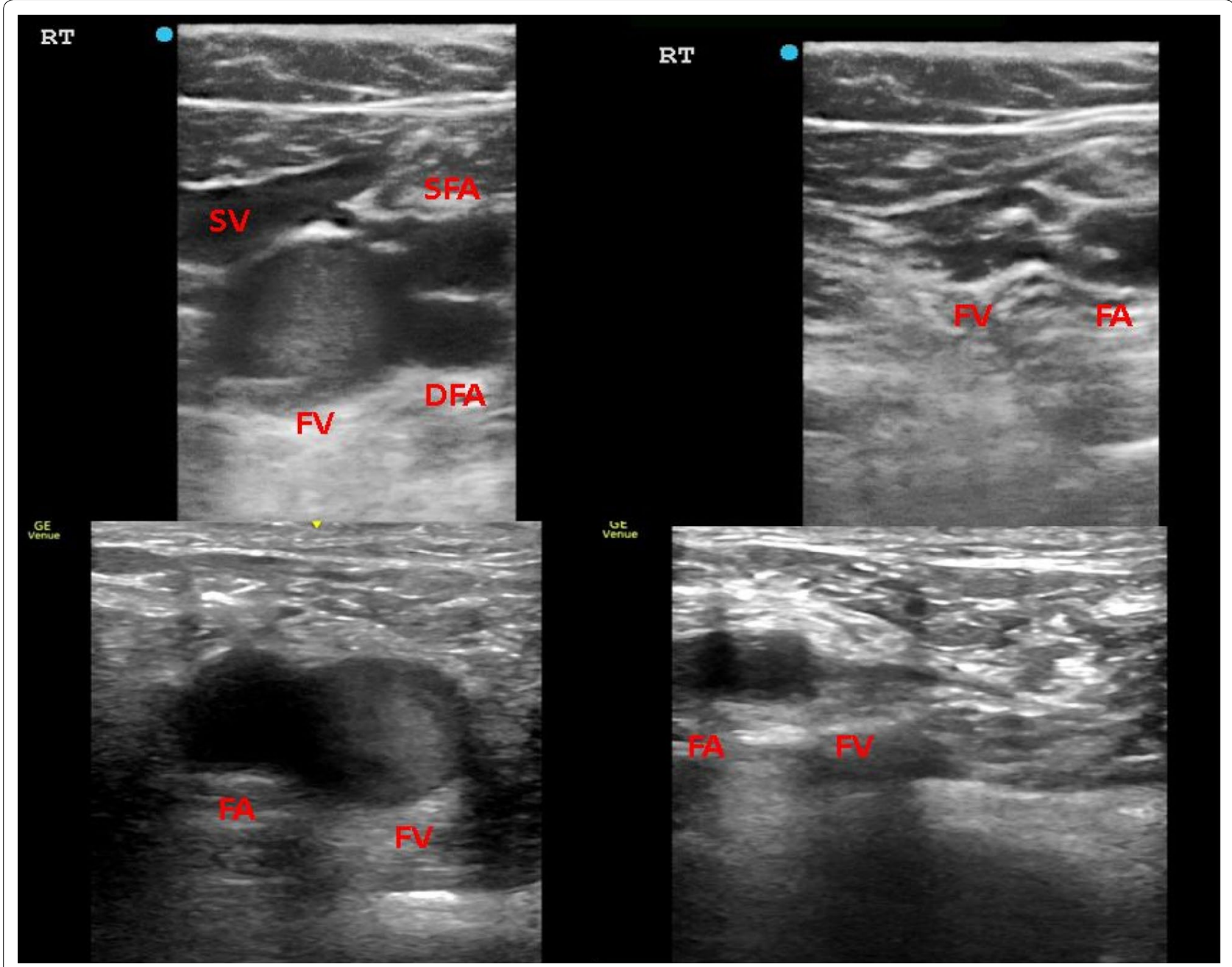

Fig. 1 The Top image shows SEC in right femoral vein with no echogenicity observed in adjacent femoral artery. The vein is fully compressible ruling out DVT at the site. The bottom image again shows SEC in fully compressible right femoral vein, with no echogenicity in adjacent femoral artery. SV saphenous vein, FV femoral vein, FA femoral artery, SFA superficial femoral artery, DFA deep femoral artery

\section{Electronic supplementary material}

The online version of this article (https://doi.org/10.1007/s00134-020-06094-3) contains supplementary material, which is available to authorized users.

\section{Author details}

${ }^{1}$ Department of Critical Care Medicine, Respiratory Institute, Cleveland Clinic, 9500 Euclid Ave, Cleveland, OH 44195, USA. ${ }^{2}$ Lerner School of Medicine of Case Western Reserve University, Cleveland, $\mathrm{OH}$, USA.

\section{Acknowledgements}

We also acknowledge Dr. Nirupama Mulherkar, Phd for proofreading of this manuscript.

\section{Funding}

The authors have no financial support to report for this manuscript. This research did not receive any specific grant from funding agencies in the public, commercial, or not-for-profit sectors.

Institutional IRB approval obtained. IRB\# 20-398

\section{Compliance with ethical standards}

\section{Conflicts of Interest}

On behalf of all authors, the corresponding author states that there is no conflict of interest.

\section{Publisher's Note}

Springer Nature remains neutral with regard to jurisdictional claims in published maps and institutional affiliations.

Accepted: 8 May 2020

Published online: 27 May 2020 


\section{References}

1. Helms J, Tacquard C, Severac F et al (2020) High risk of thrombosis in patients in severe SARS-CoV-2 infection: a multicenter prospective cohort study. Intensive Care Med. https://doi.org/10.1007/s00134-020-06062-x

2. Klok FA, Kruip MJHA, van der Meer NJM et al (2020) Incidence of thrombotic complications in critically ill ICU patients with COVID-19. Thromb Res. https://doi.org/10.1016/j.thromres.2020.04.013

3. Llitjos JF, Leclerc M, Chochois C et al (2020) High incidence of venous thromboembolic events in anticoagulated severe COVID-19 patients. J Thromb Haemost. https://doi.org/10.1111/jth.14869
4. Kory PD, Pellecchia CM, Shiloh AL et al (2011) Accuracy of ultrasonography performed by critical care physicians for the diagnosis of DVT. Chest. https://doi.org/10.1378/chest.10-1479

5. Jensen CT, Chahin A, Amin VD et al (2017) Qualitative slow blood flow in lower extremity deep veins on doppler sonography: quantitative assessment and preliminary evaluation of correlation with subsequent deep venous thrombosis development in a tertiary care oncology center. J Ultrasound Med Off J Am Inst Ultrasound Med 36:1867-1874. https://doi. org/10.1002/jum.14220 